\title{
Persistent Homology applied to location problems
}

\author{
Driss Bennis ${ }^{1, a}$, Fouad Gharib ${ }^{1, b}$ and Ghita Lebbar ${ }^{1, c}$ \\ ${ }^{1}$ Faculty of Sciences, Research Center CeReMAR, Mohammed V University in Rabat, Morocco
}

\begin{abstract}
Different approaches to solve location problems in transport and logistics have been developed in the literature. This article introduces a new approach using the concept of persistent homology which has been proved to be an efficient method in topological data analysis; and has been served as an alternative new tool in many and various research areas such as image processing, material science and biological systems. Precisely, inspired by the notions of the first homology groups and the persistent homology which mainly describe the behaviour of connectivity relation between elements during a filtration of specific topological spaces; we develop a new method and approach for the treatment of facility location-network design problems.
\end{abstract}

\section{Introduction}

The concept of persistence is first introduced by Edelsbrunner, Letscher, and Zomorodian, in [6]; then refined by Carlsson and Zomorodian in [21]. It was used to provide a rigorous response to the following problem: For a parameterized family of spaces, those topological features which persist over a significant parameter range are to be considered as signal with short-lived features as noise (see [7]). Since then, persistent homology has gathered an enormous attention as a fundamental tool in Topological Data Analysis (TDA). It has proved utility and significance to solve different problems in several domains such as biology, image processing and sensor networks (see, for instance, the papers $[1,13,16,19]$ ). For a general background on persistent homology, worth to mention the book by Zomorodian [20], survey by Edelsbrunner, Harer [5] and the survey by Ghrist [7]. The mentioned authors offer an introduction to persistent homology which can be useful for mathematical researchers based on topology.

The main interest of our work is related to location decision using persistent homology. Location decision is a critical element in transport planning. In general, decision makers are asked to select a site (location) among several existing sites (cities, district, airports, ...) on a given network in order to satisfy a number of customers while considering some constraints. Several researchers have developed different mathematical models for location decision which are mainly based on the classical optimisation methods. The present paper provides a new treatment of location problems using the concept of persistent homology. Therefore, for a practical understanding, the second section presents some basic notions and background related to persistent homology. Section 3 highlights the problem related to transport design. In section 4 , we will focus on our contribution, based on a location decision issue and the adopted approach using persistent homology to solve the problem.

\section{Mathematical background}

In this section, we give a short presentation on some basic notions concerning simplicial complexes, simplicial homology and persistent homology (more details can be found in $[5,7,20])$.

\subsection{Simplicial complexes}

An abstract simplicial complex or simply a simplicial complex is a set $\mathrm{K}$ together with a collection $\mathrm{S}$ of subsets of $\mathrm{K}$ which satisfies the two following conditions:

- $S$ contains all singletons $\{\mathrm{v}\}$ with $\mathrm{v} \in \mathrm{K}$.

- $S$ is closed under subsets: if $\tau \subseteq \sigma$ and $\sigma \in \mathrm{S}$, then $\tau \in \mathrm{S}$.

An element $\sigma$ of $S$ of cardinal a positive integer $n$ will be called an (n-1)-simplex or simply a simplex. So, 0simplices are the singletons which are usually called points; and 1-simplices consist of two elements of $\mathrm{K}$ called edges. There are also the 2-simplices called triangles and so on. In fact, any simplicial complex can be represented in a real affine space using points, edges,

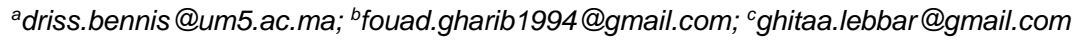


triangles and so on. Thus, using the induced topology on this geometric representation, one can talk about the connected components of a simplicial complex. Namely, this notion is of great interest in simplicial homology theory. Next section shows that the number of connected components of a simplicial complex is the dimension of some kind of vector spaces. Several examples of simplicial complex can be found in the literature. The most useful ones are geometric simplicial complexes, complexes constructed from graphs, complexes constructed from a covering of a geometric space (Nerve of a covering).

\subsection{Simplicial homology}

Simplicial homology is a powerful tool for description and characterization of some topological features such as connectivity and the existence of holes. Given $\mathrm{K}$ as a simplicial complex. The $\mathbb{Z} / 2 \mathbb{Z}$ vector space generated by the $\mathrm{p}$-dimensional simplices of $\mathrm{K}$ is denoted $\mathrm{C}_{\mathrm{p}}(\mathrm{K})$. It consists of all $\mathrm{p}$-chains, which are formal sums

$$
c=\sum_{j} \gamma_{j} \sigma_{j}
$$

where the $\gamma_{j}$ are 0 or 1 (in $\mathbb{Z} / 2 \mathbb{Z}$ ) and the $\sigma_{j}$ are $p$ simplices in $\mathrm{K}$. For a positive integer $\mathrm{p}$, consider the linear map $\partial: \mathrm{C}_{\mathrm{p}}(\mathrm{K}) \rightarrow \mathrm{C}_{\mathrm{p}-1}(\mathrm{~K})$, called boundary map, defined on n-simplices as follows: For every p-simplex $\sigma$, $\partial(\sigma)$ is the formal sum of the (p-1)-dimensional faces (i.e., subsets of $\sigma$ of cardinal p). Thus, the boundary of the chain $c$ (i.e., $\partial(\mathrm{c}))$ is obtained by extending $\partial$ linearly, and so

$$
\partial(\mathrm{c})=\sum_{j} \gamma_{j} \partial\left(\sigma_{\mathrm{j}}\right),
$$

where we understand that the addition between coefficients is modulo 2, i.e.

$$
1+1=0
$$

It is not difficult to check that:

$$
\partial \circ \partial=\partial^{2}=0
$$

The p-chains that have boundary 0 are called p-cycles. They form a subspace $Z_{p}$ of $C_{p}$. The p-chains which are the boundary of $(p+1)$-chains are called $p$-boundaries and form a subspace $B_{p}$ of $C_{p}$. The fact that:

$$
\partial^{2}=0
$$

implies that $\mathrm{B}_{\mathrm{p}} \subseteq \mathrm{Z}_{\mathrm{p}}$. The quotient group

$$
\mathrm{H}_{\mathrm{p}}(\mathrm{K}):=\mathrm{Z}_{\mathrm{p}} / \mathrm{B}_{\mathrm{p}}
$$

is the $\mathrm{p}$-th simplicial homology group of $\mathrm{K}$ with $\mathbb{Z} / 2 \mathbb{Z}$ coefficients. The dimension of $\mathrm{H}_{\mathrm{p}}(\mathrm{K})$ is called the p-th Betti number or simply the $\mathrm{p}$-Betti number of $\mathrm{K}$ and it is denoted $\beta_{\mathrm{p}}(\mathrm{K})$ or just $\beta_{\mathrm{p}}$.

Overall, the homology groups describe spaces through their Betti numbers $\beta_{\mathrm{p}}$. Precisely, $\beta_{0}$ refers to the number of connected components of the given simplicial complex, $\beta_{1}$ refers to the number of holes of the simplicial complex.

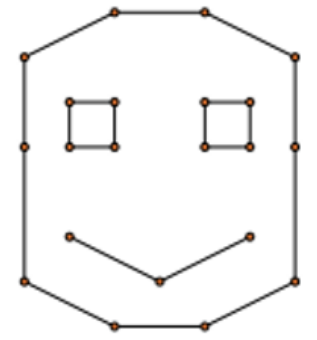

(a)

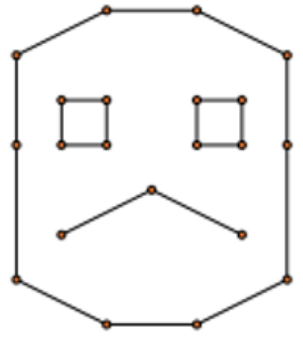

(b)
Figure 1: Two simplicial complexes with similar Betti numbers: $\beta_{0}=4$ and $\beta_{1}=3$.

\subsection{Persistent homology}

Persistent homology is considered as a powerful tool in topological shape analysis which describes the changes in homology that occur to an object with respect to a given parameter.

For a simplicial complex $\mathrm{K}$, a filtration $\mathrm{F}$ of $\mathrm{K}$ is a finite sequence of complexes:

$$
\text { F: } \emptyset=\mathrm{K}_{0} \subseteq \mathrm{K}_{1} \subseteq \cdots \subseteq \mathrm{K}_{\mathrm{m}}=\mathrm{K}
$$

The idea of persistent homology consists of, instead of examining the homology $\mathrm{H}_{\mathrm{i}}(\mathrm{K} ; \mathrm{F})$ of each individual term $\mathrm{K}_{\mathrm{i}}$ of the filtration $\mathrm{F}$, the iterated inclusions are examined:

$$
\mathrm{h}: \mathrm{H}_{\mathrm{i}}(\mathrm{K} ; \mathrm{F}) \rightarrow \mathrm{H}_{\mathrm{j}}(\mathrm{K} ; \mathrm{F}) \text { for all } \mathrm{i}<\mathrm{j} \text {. }
$$

These maps reveal which features persist. For a clear understanding, let us give a simple example visualised in the above figures:

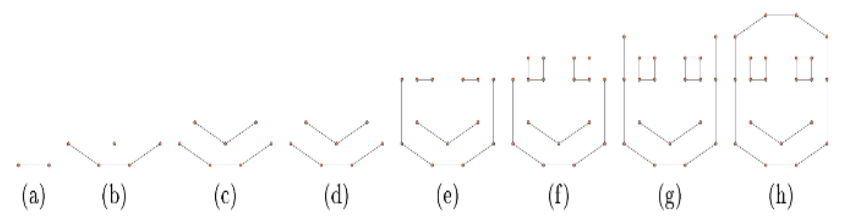

Figure2: Filtration $\mathrm{F}_{1}$ of Happy face $\mathrm{P}_{1}$

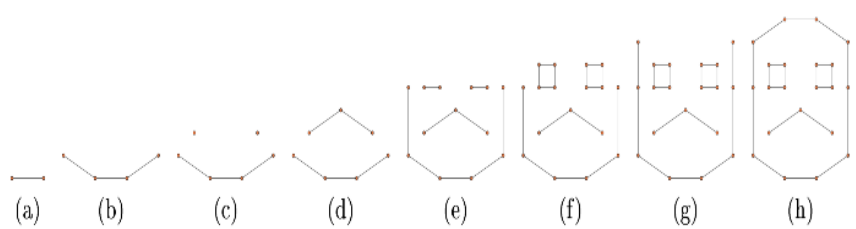

Figure 3: Filtration $F_{2}$ of Sad face $\mathrm{P}_{2}$ 
Although both pictures $\mathrm{P}_{1}$ and $\mathrm{P}_{2}$ are different, they have identical betti numbers. Persistent homology reveals the changes in homologies in the given filtration and prove the difference between both objects. Usually, the parameter intervals arising from the basis for $\mathrm{H} *(\mathrm{~K} ; \mathrm{F})$ are visualised in the form of a barcode. Precisely, a barcode is a graphical representation of $\mathrm{Hk}(\mathrm{K} ; \mathrm{F})$ as a collection of horizontal line segments in a plane whose horizontal axis corresponds to the parameter and whose vertical axis represents an arbitrary) ordering of homology generators (i.e., generators of $\mathrm{H} *(\mathrm{~K} ; \mathrm{F})$ ). For example, the barcode corresponding to the filtration $F_{1}$ above is of the form:

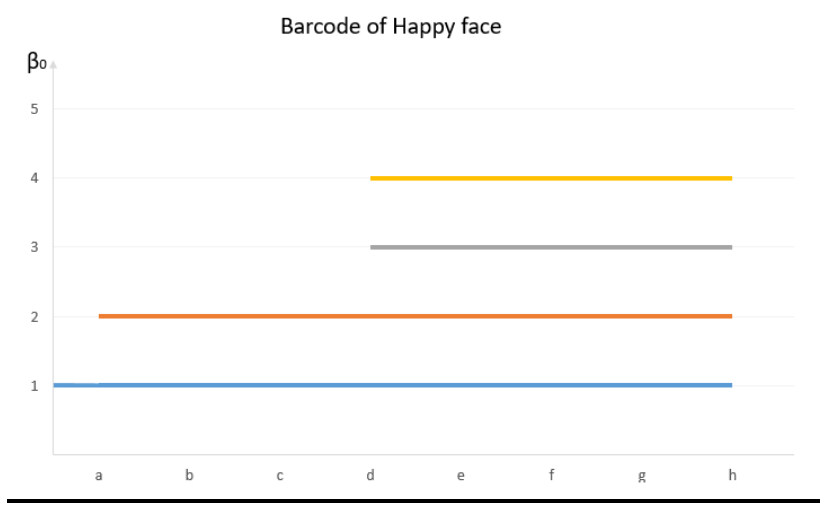

Figure 4: Barcode of happy face

And the barcode corresponding to the filtration $\mathrm{F}_{2}$ is of the form:

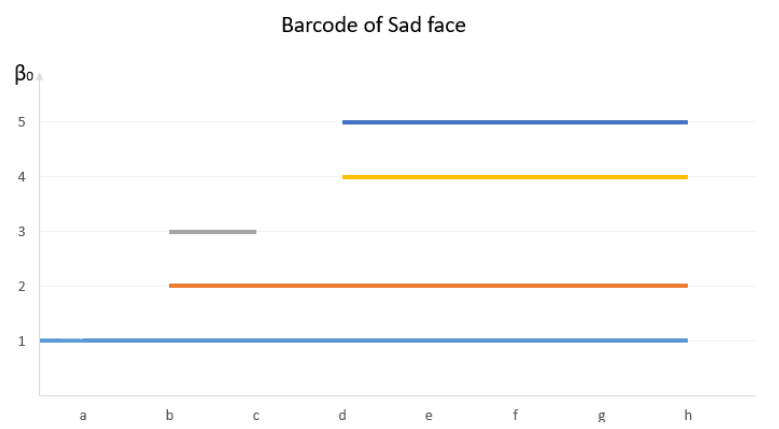

Figure 5: Barcode of sad face

The two barcodes illustrate clearly the difference.

\section{Location decision}

Location decisions have been analysed since 1909 by Weber [18] who formulated a theory to locate an industry with a minimum transportation cost of raw and final products.
Mainly, the acquisition of a new facility within a network is a costly, time-sensitive target. Hence, experts on transport design focus on solving the following issues:

$>$ The optimal choice of nodes to locate facilities

$>$ Minimize the travel cost

$>$ Reduce travel time

$>$ Optimise networks: Infrastructure (roads, bridges, tunnels...), telecommunication (cables) water pipes, electricity cables.

Hence, it can be understood that the choice of location is not arbitrary but requires strategic and expert planners which are challenged by several factors. As an aid for making decisions, the domain of facility location provides tools and methods for finding optimal locations using mathematical models with respect to quantifiable factors. O'Kelly [11] is one of the researchers who proposed a model for hub facility location based on the optimisation method.

\section{Persistent Homology as a tool for facility location}

This paper reports a new formulation for facility location by developing algorithms inspired from persistent homology. We treat the problem given in [17] through our method which can be applied in different contexts.

The starting point will be a given network consisting of $\mathrm{n}$ districts annotated by $\left(\mathrm{A}_{1}, \mathrm{~A}_{2}, \mathrm{~A}_{3}, \ldots, \mathrm{A}_{\mathrm{n}}\right)$ (nodes) in an area of a city with information about distances $d_{i, j}$ between districts $A_{i}$ and $A_{j}$, and numbers of population $n_{i}$ in each node $A_{i}$. A chain of supermarkets decided to open a facility within the districts and they need to examine the most optimum location to serve the whole area.

\subsection{Method}

The proposed method will follow a procedure which will be explained as follows:

First, it is necessary to fix some nodes as candidates where the facility can be located. The choices are based on transport design criteria which are adopted by planning experts. Then, for a fixed candidate $A_{i}$, ponderate each other node $A_{j}$ by the following coefficient which reflect the "importance" of the node $A_{j}$ with respect to $A_{i}$ (see below for the explanation):

$$
X_{j}^{i}=\frac{1}{n_{i}} \frac{d_{i, j}}{n_{j}}
$$

In fact, fixing two candidates $A_{m}$ and $A_{n}$, and 
consider two nodes $A_{e}$ and $A_{f}$. We discuss the following particular situations:

- If we suppose that our network consists only of three nodes $A_{m}, A_{n}$ and $A_{e}$. Then, $A_{m}$ is the optimal solution if $X_{e}^{m} \leq X_{e}^{n}$. Assume, for example, $\mathrm{d}_{\mathrm{e}, \mathrm{m}}=\mathrm{d}_{\mathrm{e}, \mathrm{n}}$, the inequality $\frac{1}{n_{m}} \leq \frac{1}{n_{n}}$ can be explained by the phrase : the bigger is $n_{m}$, the more attractive is $A_{m}$. On the other hand, if $n_{m}=$ $\mathrm{n}_{\mathrm{n}}, \mathrm{d}_{\mathrm{e}, \mathrm{m}} \leq \mathrm{d}_{\mathrm{e}, \mathrm{n}}$ explain the chose of $\mathrm{A}_{\mathrm{m}}$ as the optimal solution.

- Now suppose that our network consists only of four nodes $A_{m}, A_{n}, A_{e}$ and $A_{f}$. Then, $A_{m}$ will be the optimal solution if:

$$
\frac{X_{e}^{m}+X_{f}^{m}}{2} \leq \frac{X_{e}^{n}+X_{f}^{n}}{2}
$$

For example, if $n_{m}=n_{n}$ and $n_{e}=n_{f}$, the means above can be reduced to the means of distances. So clearly the optimal solution corresponds to the smallest mean. Also, the ratios $\frac{1}{n_{e}}$ and $\frac{1}{n_{f}}$ are there since the number of clients has to affect the number in some sense.

Basing on the above observations we construct a complex $\mathrm{K}_{\mathrm{m}, \mathrm{r}}$ associated to a candidate $\mathrm{A}_{\mathrm{m}}$ and for $\mathrm{a}$ fixed strictly positive real $r$ as follows (we focus only 0 simplices and 1-simplices):

- The 0-simplices are all the points $A_{j}$ which are different from $A_{m}$.

- Two nodes $A_{i}$ and $A_{j}$ is related by an edge (i.e., $\left\{A_{i}\right.$, $\left.\mathrm{A}_{\mathrm{j}}\right\}$ is a 1-simplex) if $\frac{x_{i}^{m}+x_{j}^{m}}{2} \leq r$.

Clearly, for $r<r^{\prime}, \mathrm{K}_{\mathrm{m}, \mathrm{r}} \subseteq \mathrm{K}_{\mathrm{m}, \mathrm{r}^{\prime}}$. This means that we can construct a filtration $\mathrm{K}_{\mathrm{m}, \mathrm{r} 1} \subseteq \cdots \subseteq \mathrm{K}_{\mathrm{m}, \mathrm{rs}}$, where $r 1, \ldots, r s$ is an increasing sequence of strictly positive real numbers. We choose such a sequence in the way that at the last real all complexes (for all candidates) have one connected component (i.e., all nodes are connected). In fact, passing from a complex to the next one in a filtration we obtain more edges (the nodes will be "more" connected) and then less connected component (the provided sequence of 0 -Betti numbers decreases). Then, we compare between the sequences of 0 -Betti numbers. The one which reaches 1 at the earliest time is the most attractive between all the other ones and then can be adopted as a solution following this approach. This can be seen easily when comparing the provided barcodes.

\subsection{Algorithms}

There are softwares which deal with the computation of persistent homology in various context. In our context we made our own programs and so all calculation is carried out using the $\mathrm{C}++$ programming by developing the following algorithms. Indeed, to determine the 0-Betti number of a complex associated to one of the candidates, we follow a procedure of three steps as follows:

Fixe a candidate $A_{i}$ and a real number $r$.

We need at first an algorithm to calculate all $X_{j}^{i}$.

\section{Algorithm 1}

$$
\begin{aligned}
& \text { For } \mathrm{j}=1, \mathrm{j} \neq \mathrm{i} \text { to } \mathrm{n} \text { do } \\
& X_{j}^{i}=\frac{1}{n_{i}} \frac{\mathrm{dij}}{n_{j}} ;
\end{aligned}
$$

End for

Then, we construct the simplicial complex $\mathrm{K}_{\mathrm{i}, \mathrm{r}}$ associated to $r$ using the following algorithm.

\section{Algorithm 2}

$$
\begin{array}{ll}
\text { 1. } & \mathscr{C}=\left\{\left\{\mathrm{A}_{\mathrm{j}}\right\} / \mathrm{j} \in\{1, \ldots, \mathrm{n}\} \text { and } \mathrm{j} \neq \mathrm{i}\right\} \\
\text { 2. } & \text { For } \mathrm{j}=1, \mathrm{j} \neq \mathrm{i} \text { to } \mathrm{n} \text { do } \\
\text { 3. } & \text { For } \mathrm{j}^{\prime}=1, \mathrm{j}^{\prime} \neq \mathrm{i} \text { to } \mathrm{n} \text { do } \\
\text { 4. } & \text { If }\left(\frac{1}{C_{j j^{\prime}}^{i}}<\mathrm{r}\right) \mathscr{C}=\mathscr{C} \cup\left\{\left\{\mathrm{A}_{\mathrm{j}}, \mathrm{A}_{\mathrm{j}^{\prime}}\right\}\right\} \\
& \text { where } C_{j j^{\prime}}^{i}=\frac{X_{j}^{i}+X_{j \prime}^{i}}{2} \\
\text { 5. } & \text { End for } \\
\text { 6. } & \text { End for }
\end{array}
$$

Finally, we determine and calculate the number of connected components of $\mathrm{K}_{\mathrm{i}, \mathrm{r}}$ using the algorithm beneath.

\section{Algorithm 3}

1. Initialize a matrix $M=\left(\mathrm{m}_{\mathrm{ij}}\right)_{1 \leq \mathrm{j} \leq \mathrm{n}, 1 \leq \mathrm{i} \leq \mathrm{n}}$ and $\mathrm{m}_{11}=\mathrm{A}_{1}$;

2. $\mathrm{i}=0$;

3. While $(\mathrm{n} \neq 0)$

4. $\mathrm{j}=0, \mathrm{k}_{1}=0$;

5. While $\left(m_{i k_{1}} \neq \varnothing\right)$

6. $\quad \mathrm{k}_{2}=0, \mathrm{~A}=m_{i k_{1}}$;

7. While $\left(\mathrm{k}_{2} \neq \mathrm{n}\right.$, and $\left.A_{k_{2}} \notin \mathrm{M}\right)$

8. $\quad$ if $\left(\left\{A_{k_{2}}, A\right\}\right.$ is 1 -simplex

then

9.

10.

11.

$$
\begin{gathered}
\mathrm{j}++; \\
\mathrm{m}_{\mathrm{ij}}=A_{k_{2}} ;
\end{gathered}
$$

end if 

12.
$\mathrm{k}_{2}++$;
13.
end While
$14 . \quad \mathrm{k}_{1}++$;
15. end While
16. $\mathrm{s}=$ the number of boxes filled in the $\mathrm{i}^{\text {th }}$ line of the Matrix M;
17. $\mathrm{n}=\mathrm{n}-\mathrm{s}$;
18. End While
19. $\beta_{0}=$ The number of lines containing at least one point;
20. Return $\beta_{0}$;

\subsection{Example}

Suppose that we have a network consisting of 8 districts annotated by $\left(A_{1}, A_{2}, A_{3}, \ldots, A_{8}\right)$ (eight nodes; see [17]):

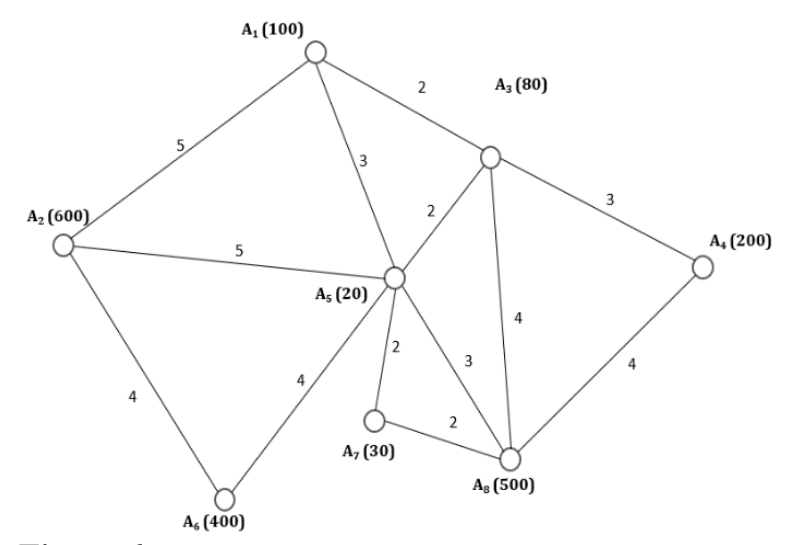

Figure 6: Network of 8 districts

We Fix three candidates $A_{2}, A_{5}$ and $A_{8}$. Then, we construct the associated barcode for each candidate.

Below, the obtained complexes in a filtration associated to $A_{2}$. We used the following sequence of real numbers: $r_{1}=1 / 5 ; r_{2}=1 / 4 ; r_{3}=2 / 7 ; r_{4}=1$.

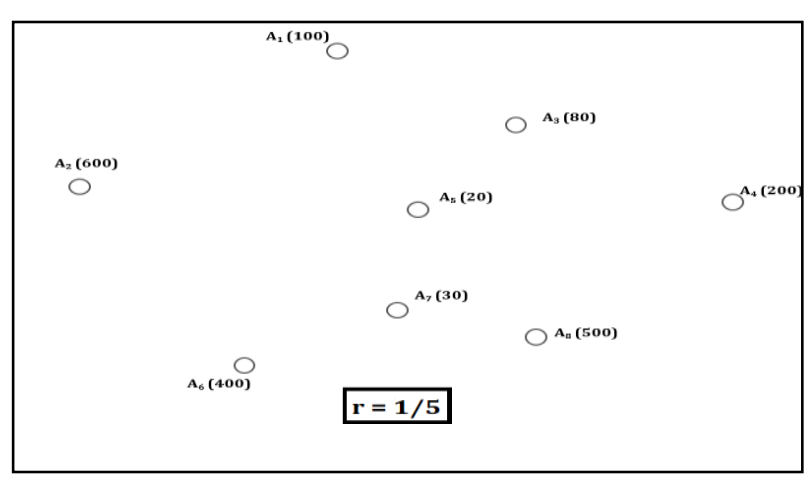

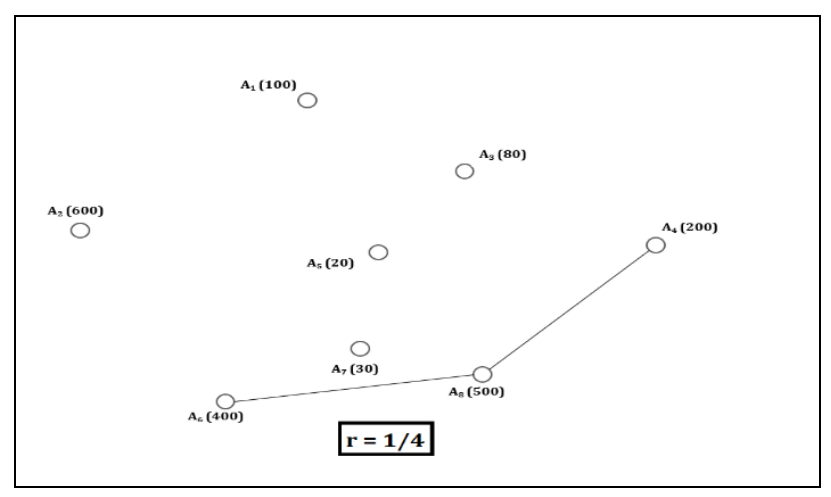

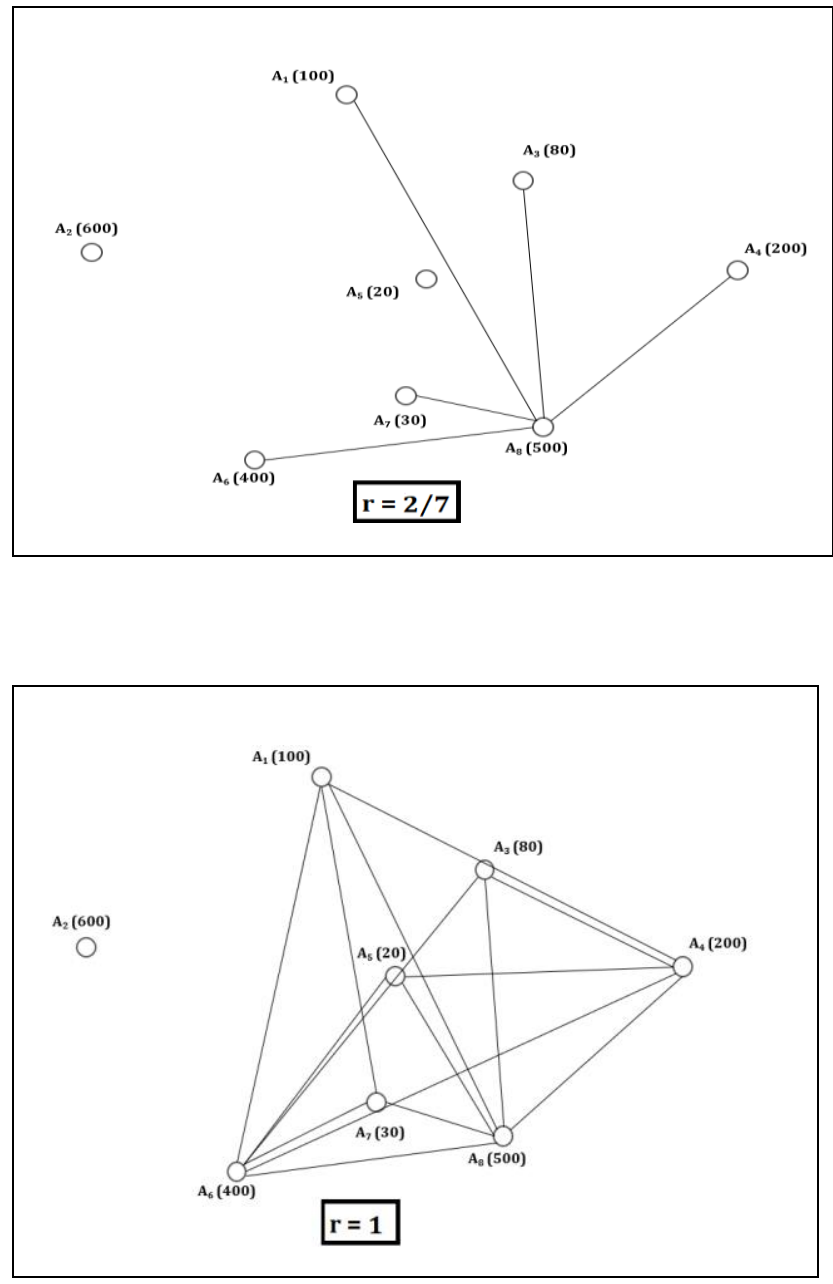

Then we obtain the following barcodes:

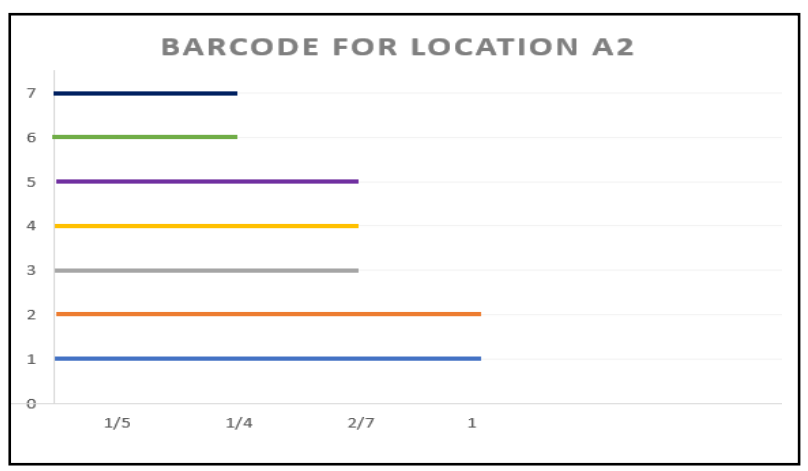



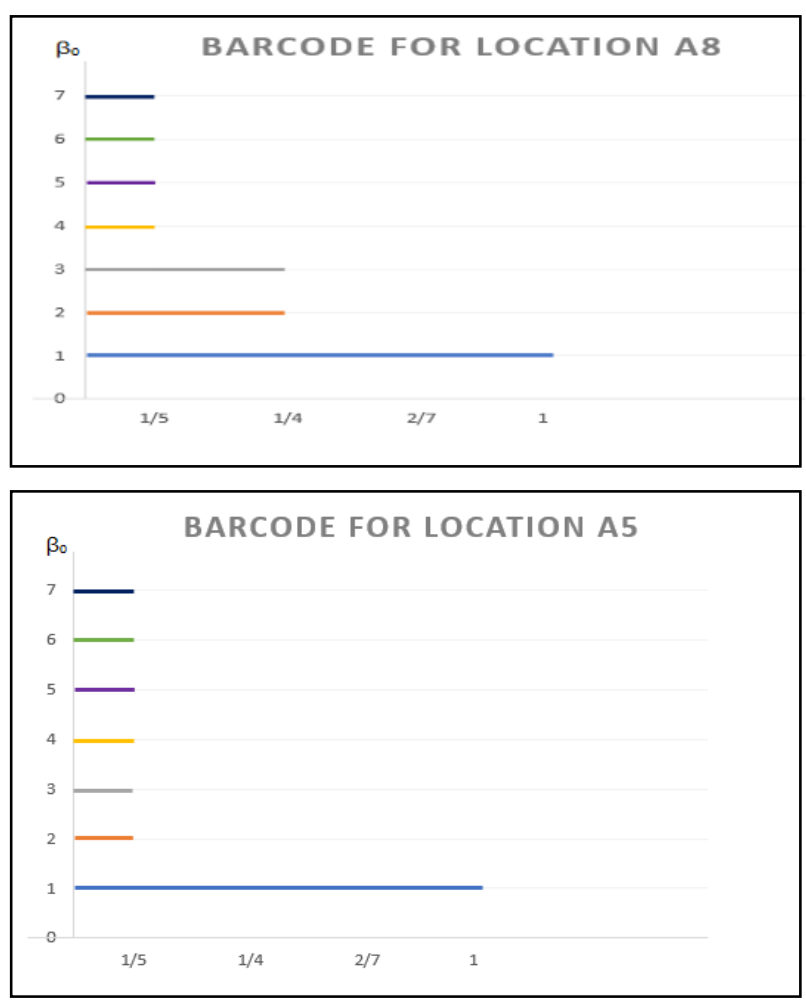

Finally, one can observe clearly that the $\mathrm{A}_{5}$ is the desired solution.

\section{Conclusion}

We have applied the proposed method to an example of eight districts; which has provided a successful location decision. Thus, this article is the first significant step made to solve location problems based on the concept of persistent homology. It will certainly attract the attention of several researchers. We expect further research to develop the solution for more complex situations with multiple parameters and for different types of location problems.

\section{References}

1. P. Bendich, J. S. Marron, E.Miller, A. Pieloch and S. Skwerer. "Persistent homology analysis of brain artery trees." Ann. Appl. Stat. 10 (2016): 198-218.

2. C. Cocking, Solutions to facility location-network design problems; Doctoral thesis at University of Heidelberg (2008).

3. Z. Drezner, G.O. Wesolowsky. "Network design: selection and design of links and facility location." Transp. Res. 37 (2003): 241-256.

4. H. Edelsbrunner, J. Harer, Computational Topology An introduction; American Mathematical Society 26 (2009).

5. H. Edelsbrunner, J. Harer. "Persistent homology - a survey." Surveys on Discrete and Computational
Geometry. Twenty Years Later, Contemporary Mathematics 453 (2008): 257-282.

6. H. Edelsbrunner, D. Letscher, A. Zomorodian. "Topological persistence and simplification." Discrete Comput. Geom. 28 (2002): 511-533.

7. R. Ghrist. "Barcodes: the persistent topology of data." Bull. Amer. Math. Soc. (N.S.), 45 (2008): 61-75.

8. A.A. Kuehn, M.J. Hamburger. "A heuristic program for locating warehouses." Manag. Sci. 9 (1963).

9. T.L. Magnanti, R.T. Wong. "Network design and transportation planning: models and algorithms." Transport. Sci. 18 (1984) : 643666.

10. M.T. Melo, S. Nickel, F. Saldanha-da-Gama. "Facility location and supply chain management - A review." Eur. J. Oper. Res. 196 (2009) 92106.

11. M.E. O'Kelly. "The location of interacting hub lacilitics." Transport Sci. 20 (1986): 92-106.

12. S.H. Owen, M.S. Daskin. "Strategic facility location: a review." Eur. J. Oper. Res. 111 (1998): 423-447.

13. J.A. Perea, J. Harer. "Sliding windows and persistence: An application of topological methods to signal analysis." Found. Comput. Math. (2013): 1-40.

14. R. Rahmaniani, A. Ghaderi. "A combined facility location and network design problem with multi-type of capacitated links." Appl Math. Model. 37 (2013): 6400-6414.

15. C. S. ReVelle, A. H. Eiselt. "Location analysis: A synthesis and survey." Eur. J. Oper. Res. 165 (2005): 1-19.

16. G. Singh, F. Memoli, G. Carlsson. "Topological methods for the analysis of high dimensional data sets and 3D object recognition." Eurographics Symposium on PointBased Graphics, eds Botsch M, Pajarola R (Eurographics Association, Geneva), (2007) 91100.

17. J. P. Škrinjar, N.Brnjac, D. Žgaljić. "Location problems in transport network." J. Mar. Res. 28 (2014): 94-98.

18. C. J. Friedrich, Alfred Weber's Theory of the Location of Industries. University of Chicago Press (1929).

19. K. Xia, Z. Li, L. Mu. «Multiscale persistent functions for biomolecular structure characterization." Perprint, available at arXiv:1612.08311 (2016).

20. A.J. Zomorodian, Topology for computing, Cambridge University Press (2005).

21. A.J. Zomorodian, G. Carlsson. "Computing Persistent Homology." Discrete Comput. Geom. 33 (2005): 249-274. 